\title{
Nicotinamide riboside exerts protective effect against aging- induced NAFLD-like hepatic dysfunction in mice
}

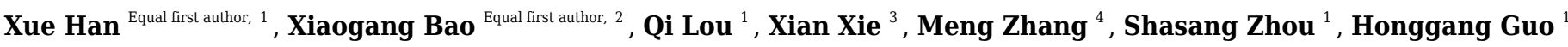 \\ , Guojun Jiang ${ }^{\text {Corresp., } 5}$, Qiaojuan Shi ${ }^{\text {Corresp. } 1}$ \\ ${ }^{1}$ Laboratory Animal Center, Zhejiang Academy of Medical Sciences, Hangzhou, Zhejiang Province, China \\ 2 Department of Orthopedic Surgery $\square$ Spine Center, Changzheng Hospital, Second Military Medical University, Shanghai, China \\ 3 Hospital of Stomatology, Zhejiang University School of Medicine, Hangzhou, Zhejiang Province, China \\ 4 Department of Clinical Laboratory, Tongde Hospital of Zhejiang Province, Hangzhou, Zhejiang Province, China \\ 5 Zhejiang Xiaoshan Hospital, Hangzhou, Zhejiang Province, China \\ Corresponding Authors: Guojun Jiang, Qiaojuan Shi \\ Email address: jguojun999@163.com, shiqiaojuan@163.com
}

Background \& Aims. Aging is one of the risk factors of non-alcoholic fatty liver disease (NAFLD). Yet, the mechanism underlying the aging-associated NAFLD-like syndrome is not fully understood. Nicotinamide adenine dinucleotide (NAD), a ubiquitous coenzyme, has protective effects against aging. Here, we investigated the actions of NAD precursors nicotinamide riboside (NR) on the development of aging-induced NAFLD. Methods. NR supplemented food ( $2.5 \mathrm{~g} / \mathrm{kg}$ food) was applied to aged mice for three months while normal chow to the other groups. Body weight, food intake, liver weight and fat pat mass were measured. The serum concentrations of lipid content, alanine aminotransferase (ALT), aspartate aminotransferase (AST) and NAD were determined by biochemical assays. Pathological assessment and immunohistochemistry analysis of hepatic tissues were used to evaluate the effect of NR on NAFLD development and inflammatory infiltration. Results. NR repletion significantly reduced fat pat mass in aged mice, while not altered the body weight, food intake, and liver weight. NR repletion significantly rescued the NAD reduction in aged mice. The total cholesterol and triglyceride levels could be lowered by NR repletion in aged mice. The AST level was also significantly reduced by NR repletion in aged group, while the ALT level lowered but without significance. Notably, moderate NAFLD phenotypes, including steatosis and hepatic fibrosis could be markedly corrected by NR repletion. In addition, Kupffer cells accumulated and inflammatory infiltration could also be remarkably reversed by NR repletion in aged mice. Conclusion. Aging was associated with NAFLD-like phenotypes in mice, which could be reversed by oral NR repletion. Therefore, oral NR uptake might be a promising strategy to halt the progression of NAFLD. 
1 Nicotinamide riboside exerts protective effect against aging-

2 induced NAFLD-like hepatic dysfunction in mice

3 Xue Han ${ }^{1, *}$, Xiaogang $\mathrm{Bao}^{2, *}$, Qi Lou ${ }^{1}$, Xian Xie ${ }^{4}$, Meng Zhang ${ }^{5}$, Shasang Zhou ${ }^{1}$, Honggang

4 Guo $^{1}$, Guojun Jiang ${ }^{3, \#}$, Qiaojuan Shi ${ }^{1, \#}$

$5 \quad{ }^{1}$ Laboratory Animal Center, Zhejiang Academy of Medical Sciences, Hangzhou, Zhejiang

6 Province, China

$7 \quad 2$ Department of Orthopedic Surgery, Spine Center, Changzheng Hospital, Second Military

8 Medical University, Shanghai, China

$9 \quad{ }^{3}$ Zhejiang Xiaoshan Hospital, Hangzhou, Zhejiang Province, China

$10{ }^{4}$ Hospital of Stomatology, Zhejiang University School of Medicine, Hangzhou, Zhejiang

11 Province, China

12 Department of Clinical Laboratory, Tongde Hospital of Zhejiang Province, Hangzhou, Zhejiang

13 Province, China

$14{ }^{*}$ These authors contributed equally to this work.

16 Corresponding Author:

17 Qiaojuan Shi ${ }^{1}$

18182 Tianmushan Road, Hangzhou, Zhejiang Province, 310013, China

19 Email address: shiqiaojuan@163.com

20 Co-corresponding author:

21 Guojun Jiang ${ }^{3}$

22728 Yucai North Road, Hangzhou, Zhejiang Province, 311200, China

23 Email address: jguojun999@163.com 


\section{Abstract}

35 Background \& Aims. Aging is one of the risk factors of non-alcoholic fatty liver disease 36 (NAFLD). Yet, the mechanism underlying the aging-associated NAFLD-like syndrome is not 37 fully understood. Nicotinamide adenine dinucleotide (NAD), a ubiquitous coenzyme, has 38 protective effects against aging. Here, we investigated the actions of NAD precursors 39 nicotinamide riboside (NR) on the development of aging-induced NAFLD.

40 Methods. NR supplemented food $(2.5 \mathrm{~g} / \mathrm{kg}$ food) was applied to aged mice for three months 41 while normal chow to the other groups. Body weight, food intake, liver weight and fat pat mass 42 were measured. The serum concentrations of lipid content, alanine aminotransferase (ALT), 43 aspartate aminotransferase (AST) and NAD were determined by biochemical assays.

44 45 Pathological assessment and immunohistochemistry analysis of hepatic tissues were used to evaluate the effect of NR on NAFLD development and inflammatory infiltration.

Results. NR repletion significantly reduced fat pat mass in aged mice, while not altered the body weight, food intake, and liver weight. NR repletion significantly rescued the NAD reduction in aged mice. The total cholesterol and triglyceride levels could be lowered by NR repletion in aged mice. The AST level was also significantly reduced by NR repletion in aged group, while the ALT level lowered but without significance. Notably, moderate NAFLD phenotypes, including steatosis and hepatic fibrosis could be markedly corrected by NR repletion. In addition, Kupffer cells accumulated and inflammatory infiltration could also be remarkably reversed by NR repletion in aged mice.

Conclusion. Aging was associated with NAFLD-like phenotypes in mice, which could be reversed by oral NR repletion. Therefore, oral NR uptake might be a promising strategy to halt the progression of NAFLD.

Subjects Gastroenterology and Hepatology, Pharmacology

Keywords Non-alcoholic fatty liver disease, nicotinamide adenine dinucleotide, nicotinamide riboside, aged mice, inflammatory infiltration 
64

65

66

67

68

69

70

71

72

73

74

75

76

77

78

79

80

81

82

83

84

85

86

87

88

89

90

Peer) reviewing PDF | (2019:04:36912:1:2:CHECK 22 Jul 2019) 


\section{Introduction}

92 Non-alcoholic fatty liver disease (NAFLD), is a metabolic disorder characterized by imbalanced

93 lipid metabolism and fatty acid accumulation in the liver. And this complex condition involves

94 the progression, from simple steatosis to inflammation (nonalcoholic steatohepatitis), and then to 95 severe fibrosis and hepatocellular carcinoma, which are the major predictors of death in patients 96 with NAFLD (Romeo, 2019). Besides, NAFLD is widely considered as hepatic manifestation of 97 metabolic syndrome. NAFLD is associated with type 2 diabetes mellitus, obesity and insulin 98 resistance, which are the main feature of metabolic syndrome (Wan and $\mathrm{Xu}, 2016$ ).

Aging is a physiological process associated with multiple organ function decline, especially the 100 liver. Specifically, aging could trigger hepatic steatosis and progressive inflammation (Jadeja and Jones, 2019). It was found that the prevalence of the NAFLD increases markedly with older age (Lee and Kim, 2007; Amarapurkar and Kamani, 2007). A clinical research, involving 589 consecutive liver biopsies, reveals that age over 30 is an independent risk factors for liver steatosis (Lee and Kim, 2007). Morbidity and age-adjusted mortality of chronic liver diseases are often more severe in older people (Frith and Jones, 2009). The aging-related liver pathology includes hepatic morphological disorder, hepatocyte polyploidization, and the reduced mitochondrial density (Gan and Chitturi, 2011; Wu and Shen, 2019). However, the mechanisms that underlie aging-related hepatic dysfunction have not been fully elucidated. Therefore, investigation of the mechanism and exploration of the therapeutic strategies are vital for the disease management.

It is well established that aging process is featured in mitochondria dysfunction and resulted depletion of nicotinamide adenine dinucleotide (NAD) depletion (Kang and Chung, 2013; Gomes AP, Price, 2013; Andreani and Bartolacci, 2018). NAD is involved in many cellular functions, and plays a crucial role in energy metabolism (Wątroba and Dudek, 2017). The salvaging synthesis pathway is predominant in NAD synthesis in mammalian cells. Nicotinamide riboside (NR) is presumed as a NAD precursor for this salvaging pathway (Moon and Kim, 2018). Once it enters the cell, NR is converted to nicotinamide mononucleotide (NMN) by ratelimiting enzyme nicotinamide phosphoribosyltransferase (NAMPT). Then, NMN is further metabolized to NAD. Additionally, the de novo biosynthesis of NAD from trytophan is considered to be another pathway for NAD synthesis. However, the de novo biosynthesis pathway only occurred in limited cell types (Yoshino and Baur, 2018). NAD level reduction and 
122 unbalance between its synthesis and consumption is proved to be related to aging-associated 123 diseases such as Parkinson's disease (PD) (Jęśko and Wencel, 2017) and Alzheimer's disease 124 (AD) (Xie and Gao, 2019). Hence, NAD supplementation might be a promising strategy to 125 restore the cellular function. Better yet, NR is found in milk, making it possible through dietary 126 modulation (Bieganowski and Brenner, 2004). In contrast to NR, other precursors of NAD 127 biosynthesis, such as nicotinic acid (NA), nicotinamide or NMN, have been shown to be severe 128 flushing or toxin in pre-clinical trials (Bogan and Brenner, 2008; Di Stefano and Nascimento129 Ferreira, 2015). Therefore, NR was highlighted as a promising compound for the rescue of NAD 130 level. Notably, NAD complementation has been previously reported to be beneficial in other 131 conditions. Karpe et al. showed that NAD complementation improved the plasma lipid and 132 cholesterol profiles, and ameliorated metabolic disorder (Karpe and Frayn, 2004). Modulation of 133 the cellular NAD level can attenuate hepatic steatosis and inflammation in mice fed with 134 methionine-choline-deficient diet (Katsyuba and Mottis, 2018). Evidences also display that 135 replenish NR has beneficial effects on NAFLD, insulin sensitivity and AD in mice (Gariani and 136 Menzies, 2016; Cantó C and Houtkooper, 2012, Xie and Gao, 2019). In short, NAD has 137 pronounced effects on hepatic homeostasis.

138 However, the relation between NAD complementation through NR supplementation and aging139 associated hepatic dysfunction has not been investigated. In the current study, we fed the aging 140 mice with NR supplied food for 3 months. We aimed to explore the role of NR repletion on 141 aging-induced NAFLD and inflammatory infiltration in aged mice. 
153 Materials \& Methods

154 Animals

155 Female C57BL/6J mice in 3-month old and 14-month old were purchased from the Laboratory 156 Animal Center of Zhejiang Province (Hangzhou, China), and used as young mice, aged mice 157 and NR supplied aged mice respectively. All mice were maintained in an environmentally158 controlled room (12 h light-dark cycle, $20-26^{\circ} \mathrm{C}$, relative humidity $50 \%$ ), fed a standard chow 159 with free access to water. All animal experiments were performed in accordance with the 160 National Institutes of Health Guide for the Care and Use of Laboratory Animals. Procedures 161 were approved by the Institutional Animal Care and Use Committee of the Laboratory Animal 162 Center of Zhejiang Province and the experimental protocols were approved by the Ethics 163 Committee of Laboratory Animal Care and Welfare, Zhejiang Academy Medical Sciences, with 164 the proved number DLSY.2017(80).

165 NR supplementation

166 The mice were divided to three groups: (1) Young mice (Young); (2) Aged mice (Aged); and (3) 167 NR treated aged mice (Aged + NR). NR (Baikai Chemical Technology Co., Ltd, Hangzhou, 168 China) was mixed into the pellets with the concentration of $2.5 \mathrm{~g} / \mathrm{kg}$ (Zhejiang Academy of 169 Medical Sciences, Hangzhou, China). Food consumption of aged mice for ten days were 170 measured and the average food intake was estimated at $160 \mathrm{~g} / \mathrm{kg}$. According to the food intake, 171 the aged mice orally treated with NR around $400 \mathrm{mg} / \mathrm{kg} / \mathrm{day}$. The food containing NR was 172 supplied from fifteen-month old and four-month old for C57BL/6J mice and lasted for three 173 months until sacrificed. Mice in the Young and Age groups were received common food 174 correspondingly. After 3 months, mice were used for ex vivo studies.

\section{Body weight and food intake determinations}

176 Body weight changes of each group were measured at the end of NR administration. 177 Additionally, the food intake of aged mice was measured in cages every 3-5 days. The average 178 daily amount of each mouse was calculated.

179 Liver weight and fat pat mass measurements

180 Mice of three groups were euthanized by chloral hydrate $(800 \mathrm{mg} / \mathrm{kg})$ injection intraperitoneally, 181 after an overnight fasting period. Mice were transcardially perfused with $4{ }^{\circ} \mathrm{C}$ saline. The livers 182 and total fat pat were quickly removed, carefully cleaned, and blotted dry. Then the collected 
183 samples were measured carefully. The ratios between tissue mass and body weight were 184 calculated respectively.

185 Blood biochemical assays

186 Blood samples were acquired from inferior vena cava of anesthetized mice. About $0.7 \mathrm{ml}$ blood 187 was harvested. Samples were still standing at room temperature for $40 \mathrm{~min}$ and at $4{ }^{\circ} \mathrm{C}$ for $2 \mathrm{~h}$, 188 then followed by $3000 \mathrm{rpm}$ centrifugation for $10 \mathrm{~min}$. The supernatant was used for blood 189 biochemical index measurement. According to the manufacturer's instructions, triglyceride(TG), 190 total cholesterol (TC), alanine aminotransferase (ALT) and aspartate aminotransferase (AST) 191 contents in serum were quantified by an automatic biochemistry analyzer (Backman). Blood 192 NAD concentration was determined by a commercial NAD Quantitation Colorimetric Kit 193 (K337-100, Biovision, San Francisco, CA, USA).

\section{Histological analysis}

195 Those isolated livers, taken from the same lobe, were fixed in 4\% paraformaldehyde for 8 hours 196 and then were embedded in paraffin for histological processing. Samples were cut into thin 197 section $(5 \mu \mathrm{m})$ and stained with hematoxylin and eosin (H\&E) to assess histopathology, and Masson's trichrome for collagen evaluations. Images were obtained at 200 magnifications under the inverted phase-contrast microscope (Leica Microsystems, Wetzlar, Germany).

200 Scoring for steatosis (severity and extension) was performed in a blinded and independent 201 method by two observers as described before (Gariani and Menzies, 2016). The analysis used a 202 scale of $0-4$, where 0 referred to absent of vacuolation in the liver, 1 referred to 2 or 3 vacuoles 203 per hepatic cord per lobule, 2 referred to less than $50 \%$ of the lobule has fatty vacuolation, 3 204 referred to more than $50 \%$ of the lobule has fatty vacuolation, and 4 corresponded to nearly the 205 entire lobule has fatty infiltration. Moreover, the focal extention was referred to 1, multifocal 206 was referred to 2, and almost total diffuse was referred to 3.

\section{Immunohistochemical analysis}

208 For immunohistochemical analysis, thin sections blocked by 5\% goat serum followed by 209 incubating in specific primary antibodies. PBS was applied to wash the sections for 3 times. 210 Then, samples was stained with horseradish peroxidase-conjugated secondary antibodies and 211 visualized by substrate DAB. Images were taken with a microscope (Leica, 200×) under same 212 acquisition settings for each section. The primary antibodies were used as follow: TGF- $\beta$ 213 (MAB240-100, R\&D System, 1:600 dilution), F4/80 (LS-C96373-100, Lifespan, 1:1000 
214 dilution), CD68 (ab125212, Abcam, 1:600 dilution), IL-1ß (SRP8033, Sigma, 1:1000 dilution), 215 TNF- $\alpha$ (ab6671, Abcam, 1: 600 dilution).

\section{Statistical analyses}

217 Data were expressed as mean \pm SEM. Values from different groups were analyzed using one218 way ANOVA followed by Newman-Keuls multiple comparison test. Statistical analysis was 219 done in GraphPad Software (Prism Version 5.01). Statistical significance was considered as $P<$ 2200.05.

221

222

223

224

225

226

227

228

229

230

231

232

233

234

235

236

237

238

239

240

241

Peer] reviewing PDF | (2019:04:36912:1:2:CHECK 22 Jul 2019) 


\section{Results}

243 Changes of body weight, food intake, relative liver weight and fat pat mass in NR treated 244 aged mice

245 After 3 months of NR supplied, body weight in aged mice with NR repletion was a little lower 246 than the aged mice $(25.2 \pm 1.2$ vs $27.3 \pm 1.1, P>0.05)$, although no significant difference was 247 shown (Figure 1A). There was also no significant difference in food intake or liver to body 248 weight ratio between NR supplied aged mice and aged mice. (Figure 1B and 1C). As shown in 249 Figure 1D, ageing was sufficient to induce fat pat mass to body weight ratio increased compared 250 with young. While the ratio was greatly decreased in aged mice with NR repletion when 251 compared to the aged mice $(3.0 \pm 0.4$ vs $4.4 \pm 0.7, P<0.05)$.

\section{NR favoured lipid homeostasis and hepatic steatosis in aged mice}

253 To answer whether the NR supplied could improve the susceptibility to development of NAFLD 254 in aged mice, we used 15 months old mice with 3 months feeding of NR. The TG and TC 255 contents were significant elevated in aged mice when compared to young mice. NR supplied 256 aged mice significantly reduced both TG $(0.87 \pm 0.02$ vs $1.12 \pm 0.12, P<0.05$, Figure $2 \mathrm{~A})$ and 257 TC level $(2.31 \pm 0.23$ vs $2.99 \pm 0.19, P<0.05$, Figure $2 \mathrm{~B})$. The results were in agreement with 258 improved fat accumulation, suggesting NR protects against age-induced lipid disorders. As a 259 result, serum elevation in ALT and AST of aged mice indicated an impaired of liver. While the 260 level of AST was greatly attenuated with NR $(98.8 \pm 8.56$ vs $124.7 \pm 10.56, P<0.05$, Figure 2D) 261 without ALT (Figure 2C). These results matched by changes in NAD concentration (Figure 2E). 262 Moreover, H\&E staining presented hepatocellar irregularity shaped and severity of steatosis in 263 aged mice (Figure 2G-L). The histology score in aged mice was greatly improved by NR 264 supplied (Figure 2F). These observations suggested that aging could promote the lipid 265 accumulation and the ensuing development of NAFLD-like hepatic dysfunction. And NR was 266 demonstrated to prevent age-induced hepatic steatosis.

267 NR improved hepatic fibrosis in aged mice

268 To further determined the influence of NR on development of NAFLD in aged mice, Masson's 269 trichrome and TGF- $\beta$ staining were performed. NR weaken hepatic collagen and fibrosis, as 270 revealed by less Masson's trichrome staining and TGF- $\beta$ staining (Figure 3A-D, 3E-H).

271 NR alleviated inflammation infiltrated in liver of aged mice 
272 We investigated the influence of $\mathrm{NR}$ on hepatic inflammation in aged mice. 273 Immunohistochemical staining for F4/80 and CD68 indicated that accumulated Kupffer cells 274 were obviously reduced in liver of NR supplemented aged mice compared with aged mice 275 (Figure 4A-D, 4I). In agreement with NR-induced improvement in macrophagocyte infiltration, 276 there was also a significant down-regulation of pro-inflammatory cytokines IL-1 $\beta$ and TNF- $\alpha$ 277 expression in liver from NR supplied aged mice compared with aged mice (Figure 4E-H, 4J).

278

279

280

281

282

283

284

285

286

287

288

289

290

291

292

293

294

295

296

297

298

299 


\section{Discussion}

301 The major findings of the present study were as follows.. First, the supplementation of NR 302 ameliorated lipid homeostasis and hepatic steatosis in aged mice. Second, the supplementation of 303 NR reduced collagen deposition and hepatic fibrosis in liver from aged mice. Finally, we showed 304 that NR treatment decreased Kupffer cells infiltrating as well as lowered IL-1 $\beta$ and TNF- $\alpha$ 305 expression in liver from aged mice.

306 Prevalence of NAFLD increases dramatically with age, although this disease appears in 307 different age groups (Zhou and Li, 2019). There are strong evidences suggesting steatohepatitis 308 and fibrosis are associated with aging, which results in a higher mortality in elderly individuals 309 with NAFLD (Argo and Northup, 2009; Ooi and Mgaieth, 2018). Researchers have identified 310 several mechanisms underlying the age promotion the morbidity of NAFLD. Physiological 311 changes characterize aging may trigger the development of components of the metabolic 312 disturbance. For example, the functional decrease in the lysosomal degradative pathway of 313 autophagy appears to be remarkable in aged individual, which may encourage lipid accumulation 314 in the liver (Martinez-Lopez and Athonvarangkul, 2015; Chi and Tsai, 2019). Furthermore, the 315 level of oxidative stress, inflammation and DNA damage increase with aging, and these 316 excessive elevations have also been implicated as mediators of NAFLD pathogenesis. Previous 317 study has reported that the histological grade of steatosis similarly increased in aged mice 318 compared with young and middle mice (Fontana and Zhao, 2013). Recently, the deteriorate 319 morphology and function of livers have also been observed in natural aging rat models (Minhas 320 and Liu, 2019). In this study, our results indicated that 18-month-old C57BL/6J mice exhibited 321 an impaired lipid homeostasis including body weight gain, fat accumulation and serum TG and 322 TC increase. These mice showed great susceptibility to development of NAFLD, reflected in 323 steatosis with moderate fatty infiltration. Liver is a vital regulator of metabolism. Therefore, it is 324 important to maintain hepatic function of elderly. Nevertheless, the little data are currently 325 available in molecular mechanism for aging-related NAFLD.

326 NAD is a substrate for multiple enzymes of sirtuin family and participates in multiple cellular 327 functions, including DNA repair, energy metabolism, and regulation the activity of the sirtuins 328 by transcriptional control (Hoxhaj and Ben-Sahra, 2019). It is well-established that aging and 329 fatty liver related dysfunction leads to a pronounced effect on decline of NAD concentration in 330 liver. Fan et al. have reported that the expression of hepatic mRNA of regulating NAD 
331 biosynthesis is greatly reduced in aged mice or challenged high-fat diet (HFD) mice (Fan and 332 Cui, 2018). Additionally, this phenomenon seems to be a toxic element, providing destructive 333 actions because a shortage of NAD links ageing to progressive liver damage. The beneficial 334 effects of NAD regiment on fatty liver have been reported, for instance in a liver-specific Sirt1 335 knockout mouse (Katsyuba and Mottis, 2018) and in an enzyme-dead NAMPT transgenic mouse 336 (Zhou and Yang, 2016). Thus, supplementation NAD pool may be an attractive therapy strategy 337 for liver damage related diseases in elderly individual. Notably, NR, this vitamin B3 analog, as a 338 precursor of NAD biosynthesis, is commonly used to boost NAD pool (Jiang and Zhou, 2019). 339 Here we showed that the replenishing of NR has beneficial effect on liver of aged mice. Our 340 study demonstrated that aged mice administrated of NR (250 mg/kg/day) for 3 months improved 341 lipid disordered. Moreover, NR treatment exhibited an amelioration in hepatic steatosis and 342 fibrosis that was matched by an augmented blood NAD concentration, implying a systemic NAD 343 replenishing in aged mice.

344 An extensive body of evidence indicates that chronic inflammation contributes to the 345 degenerative changes of full-length tissues in the context of aging. Even normal brain of aged 346 individual is characterized by increased inflammation and subsequently elevated pro347 inflammatory cytokines (Frank and Barrientos, 2006). As inflammation rose by age shows a 348 reduction in adequate NAD content in brain of the murine (Braidy and Guillemin, 2011). 349 Previous study has also demonstrated that genetic blockade of NAD synthesis exerts 350 inflammatory effects on the liver reflecting by activation NLRP-3 inflammasome pathway and 351 production of IL-18 and IL-1 $\beta$ (Jiang and Zhou, 2019). The other independent group shows that 352 pharmacological inhibition of de novo NAD synthesis strengthens transcription genes involved 353 in inflammation, including Desmin and Tgfb (Katsyuba and Mottis, 2018). Intriguingly, 354 increasing the NAD concentration leads to promote pro-inflammatory cytokine synthesis by 355 activated immune cells (Van Gool and Gallí M, 2009). Consistently, decreasing the NAD pool causes innate immune disorder in aging-associated diseases (Minhas and Liu, 2019). In the present study, we found that the supplementation of NR obviously weakened Kupffer cell 358 accumulation accompanied by inhibiting expression of IL-1 $\beta$ and TNF- $\alpha$. In the context of lipid 359 accumulation, macrophages are recruited into liver and pro-inflammatory cytokines subsequently produced in liver of aged mice. This data probably is discrepancy with several previous reports, 
361 thus further evidences are still needed to confirm the relationship between NAD level and 362 inflammatory reaction.

363

364 Conclusions

365 In summary, in the present study we show that aging-related NAD deficiency causes pathologic 366 changes and inflammation infiltration in liver of aged mice. The replenishment of NAD, by 367 treated with NR, is able to protect against aging-induced hepatic steatosis, which is possibly 368 associated with an improvement in reduction of pro-inflammatory cytokines, such as IL-1 $\beta$ and 369 TNF- $\alpha$. Our study raises the possibility of NR to alleviate NAFLD-like liver injure in aged 370 individuals, suggesting the clinical advantage of NR during vitamin supplementation therapy. 371 Further investigations are warranted to treat aging-related liver diseases by NAD 372 supplementation strategy.

373

374 ACKNOWLEDGMENTS

375 This study was supported by the Natural Science Foundation of Zhejiang (Grants 376 LGJ18H310002, LQY19H090001, LY18H310009 and LQY18C040001) and the Shanghai 377 Committee of Science and Technology, China (Grant No. 15411951000 and 2018QN13).

378

379

380

381

382

383

384

385

386

387

388

389

390

391

392

393 
394

395

396

397

398

399

400

401

402

403

404

405

406

407

408

409

410

411

412

413

414

415

416

417

418

419

420

421

422

423

424

425

\section{References}

Amarapurkar D, Kamani P, Patel N, Gupte P, Kumar P, Agal S, Baijal R, Lala S, Chaudhary D, Deshpande A. 2007. Prevalence of non-alcoholic fatty liver disease: population based study. Ann Hepatol 6(3):161-163.

Andreani C, Bartolacci C, Guescini M, Battistelli M, Stocchi V, Orlando F, Provinciali M, Amici A, Marchini C, Tiano L, Orlando P, Silvestri S. 2018. Combination of Coenzyme Q10 Intake and Moderate Physical Activity Counteracts Mitochondrial Dysfunctions in a SAMP8 Mouse Model. Oxid Med Cell Longev 2018:8936251. doi $10.1155 / 2018 / 8936251$.

Argo CK, Northup PG, Al-Osaimi AM, Caldwell SH. 2009. Systematic review of risk factors for fibrosis progression in non-alcoholic steatohepatitis. $J$ Hepatol 51(2):371-9 doi 10.1016/j.jhep.2009.03.019.

Bieganowski P, Brenner C. 2004. Discoveries of nicotinamide riboside as a nutrient and conserved NRK genes establish a Preiss-Handler independent route to NAD+ in fungi and humans. Cell 117(4): 495-502.

Bogan KL, Brenner C. 2008. Nicotinic acid, nicotinamide, and nicotinamide riboside: a molecular evaluation of NAD+ precursor vitamins in human nutrition. Annu Rev Nutr 28:115-130 doi 10.1146/annurev.nutr.28.061807.155443.

Braidy N, Guillemin GJ, Mansour H, Chan-Ling T, Poljak A, Grant R. 2011. Age related changes in NAD + metabolism oxidative stress and Sirt1 activity in wistar rats. PLoS One 6(4):e19194 doi 10.1371/journal.pone.0019194.

Cantó C, Houtkooper RH, Pirinen E, Youn DY, Oosterveer MH, Cen Y, FernandezMarcos PJ, Yamamoto H, Andreux PA, Cettour-Rose P, Gademann K, Rinsch C, Schoonjans K, Sauve AA, Auwerx J. 2012. The NAD $(+)$ precursor nicotinamide riboside enhances oxidative metabolism and protects against high-fat diet-induced obesity. Cell Metab 15(6):838-47 doi 10.1016/j.cmet.2012.04.022.

Chi HC, Tsai CY, Tsai MM, Yeh CT, Lin KH. 2019. Molecular functions and clinical impact of thyroid hormone-triggered autophagy in liver-related diseases. $J$ Biomed Sci 26(1):24 doi 10.1186/s12929-019-0517-x.

Di Stefano M, Nascimento-Ferreira I, Orsomando G, Mori V, Gilley J, Brown R, Janeckova L, Vargas ME,Worrell LA, Loreto A, Tickle J, Patrick J,Webster JR, Marangoni M, Carpi FM, Pucciarelli S, Rossi F, Meng W, Sagasti A, Ribchester RR, 
426

427

428

429

430

431

432

433

434

435

436

437

438

439

440

441

442

443

444

445

446

447

448

449

450

451

452

453

454

455

456

Magni G, Coleman MP, Conforti L. 2015. A rise in NAD precursor nicotinamide mononucleotide (NMN) after injury promotes axon degeneration. Cell Death Differ 22(5):731-742 doi 10.1038/cdd.2014.164.

Fan R, Cui J, Ren F, Wang Q, Huang Y, Zhao B, Wei L, Qian X, Xiong X. 2018. Overexpression of NRK1 ameliorates diet- and age-induced hepatic steatosis and insulin resistance. Biochem Biophys Res Commun 500(2):476-483 doi 10.1016/j.bbrc.2018.04.107.

Fontana L, Zhao E, Amir M, Dong H, Tanaka K, Czaja MJ. 2013. Aging promotes the development of diet-induced murine steatohepatitis but not steatosis. Hepatology 57(3):9951004 doi 10.1002/hep.26099.

Frank MG, Barrientos RM, Biedenkapp JC, Rudy JW, Watkins LR, Maier SF. 2006. mRNA up-regulation of MHC II and pivotal pro-inflammatory genes in normal brain aging. Neurobiol Aging 27(5):717-22 doi 10.1016/j.neurobiolaging.

Frith J, Jones D, Newton JL. 2009. Chronic liver disease in an ageing population. Age Ageing 38(1):11-8 doi 10.1093/ageing/afn242.

Gan L, Chitturi S, Farrell GC. 2011. Mechanisms and implications of age-related changes in the liver: nonalcoholic Fatty liver disease in the elderly. Curr Gerontol Geriatr Res 2011:831536 doi 10.1155/2011/831536.

Gariani K, Menzies KJ, Ryu D, Wegner CJ, Wang X, Ropelle ER, Moullan N, Zhang H, Perino A, Lemos V, Kim B, Park YK, Piersigilli A, Pham TX, Yang Y, Ku CS3, Koo SI, Fomitchova A, Cantó C, Schoonjans K, Sauve AA, Lee JY, Auwerx J. 2016. Eliciting the Mitochondrial Unfolded Protein Response by Nicotinamide Adenine Dinucleotide Repletion Reverses Fatty Liver Disease in Mice. Hepatology 63(4):1190-204 doi 10.1002/hep.28245.

Gomes AP, Price NL, Ling AJ, Moslehi JJ, Montgomery MK, Rajman L, White JP, Teodoro JS, Wrann CD, Hubbard BP, Mercken EM, Palmeira CM, de Cabo R, Rolo AP, Turner N, Bell EL, Sinclair DA. 2013. Declining NAD $(+)$ induces a pseudohypoxic state disrupting nuclear-mitochondrial communication during aging. Cell 155(7): 1624-1638 doi 10.1016/j.cell.2013.11.037.

Hoxhaj G, Ben-Sahra I, Lockwood SE, Timson RC, Byles V, Henning GT, Gao P, Selfors LM, Asara JM, Manning BD. 2019. Direct stimulation of NADP+ synthesis through Aktmediated phosphorylation of NAD kinase. Science 363(6431):1088-1092 doi 10.1126/science.aau3903. 
457

458

459

460

461

462

463

464

465

466

467

468

469

470

471

472

473

474

475

476

477

478

479

480

481

482

483

484

485

486

Jadeja RN, Jones MA, Fromal O, Powell FL, Khurana S, Singh N, Martin PM. 2019. Loss of GPR109A/HCAR2 induces aging-associated hepatic steatosis. Aging 11(2):386-400 doi 10.18632/aging.101743.

Jęśko H, Wencel P, Strosznajder RP, Strosznajder JB. 2017. Sirtuins and Their Roles in Brain Aging and Neurodegenerative Disorders. Neurochem Res 42(3):876-890 doi 10.1007/s11064-016-2110-y.

Jiang R, Zhou Y, Wang S, Pang N, Huang Y, Ye M, Wan T, Qiu Y, Pei L, Jiang X, Huang Y, Yang H, Ling W, Li X, Zhang Z, Yang L. 2019. Nicotinamide riboside protects against liver fibrosis induced by $\mathrm{CCl} 4$ via regulating the acetylation of Smads signaling pathway. Life Sci pii: S0024-3205(19)30228-0 doi 10.1016/j.1fs.2019.03.064.

Kang C, Chung E, Diffee G, Ji LL. 2013. Exercise training attenuates aging-associated mitochondrial dysfunction in rat skeletal muscle: role of PGC-1 $\alpha$. Exp Gerontol 48(11):134350 doi 10.1016/j.exger.2013.08.004.

Karpe F, Frayn KN. 2004. The nicotinic acid receptor-a new mechanism for an old drug. Lancet 363(9424):1892-4 doi 10.1016/S0140-6736(04)16359-9.

Katsyuba E, Mottis A, Zietak M, De Franco F, van der Velpen V, Gariani K1, Ryu D1, Cialabrini L, Matilainen O1, Liscio P, Giacchè N, Stokar-Regenscheit N, Legouis D, de Seigneux S, Ivanisevic J, Raffaelli N, Schoonjans K, Pellicciari R, Auwerx J. 2018. De novo NAD+ synthesis enhances mitochondrial function and improves health. Nature 563(7731):354-359 doi 10.1038/s41586-018-0645-6.

Lee JY, Kim KM, Lee SG, Yu E, Lim YS, Lee HC, Chung YH, Lee YS, Suh DJ. 2007. Prevalence and risk factors of nonalcoholic fatty liver disease in potential living liver donors in Korea: a review of 589 consecutive liver biopsies in a single center. J Hepatol 47(2):239244 doi 10.1016/j.jhep.2007.02.007. Martinez-Lopez N, Athonvarangkul D, Singh R. 2015. Autophagy and aging. Adv Exp Med Biol 847:73-87 doi 10.1007/978-1-4939-2404-2_3. Minhas PS, Liu L, Moon PK, Joshi AU, Dove C, Mhatre S, Contrepois K, Wang Q, Lee BA, Coronado M, Bernstein D, Snyder MP, Migaud M, Majeti R, Mochly-Rosen D, Rabinowitz JD, Andreasson KI. 2019. Macrophage de novo NAD+ synthesis specifies immune function in aging and inflammation. Nat Immunol 20(1):50-63 doi 10.1038/s41590018-0255-3. 
487

488

489

490

491

492

493

494

495

496

497

498

499

500

501

502

503

504

505

506

507

508

509

510

511

512

513

514

515

516

517

Moon J, Kim HR, Shin MG. 2018. Rejuvenating Aged Hematopoietic Stem Cells Through Improvement of Mitochondrial Function. Ann Lab Med 38(5):395-401 doi 10.3343/alm.2018.38.5.395.

Ooi GJ, Mgaieth S, Eslick GD, Burton PR, Kemp WW, Roberts SK, Brown WA. 2018. Systematic review and meta-analysis: non-invasive detection of non-alcoholic fatty liver disease related fibrosis in the obese. Obes Rev 19(2):281-294 doi 10.1111/obr.12628.

Romeo S. 2019. Notch and Nonalcoholic Fatty Liver and Fibrosis. N Engl J Med 380(7):681683 doi 10.1056/NEJMcibr1815636.

Van Gool F, Gallí M, Gueydan C, Kruys V, Prevot PP, Bedalov A, Mostoslavsky R, Alt FW, De Smedt T, Leo O. 2009. Intracellular nad levels regulate tnf- $\alpha$ protein synthesis in a sirtuin-dependent manner. Nat Med 15(2):206-10. doi 10.1038/nm.1906.

Wan X, Xu C, Lin Y, Lu C, Li D, Sang J, He H, Liu X, Li Y, Yu C. 2016. Uric acid regulates hepatic steatosis and insulin resistance through the NLRP3 inflammasome-dependent mechanism. J Hepatol 64(4):925-32 doi 10.1016/j.jhep.2015.11.022.

Wątroba M, Dudek I, Skoda M, Stangret A, Rzodkiewicz P, Szukiewicz D. 2017. Sirtuins, epigenetics and longevity. Ageing Res Rev 40:11-19 doi 10.1016/j.arr.2017.08.001.

Wu FH, Shen CH, Luo SC, Hwang JI, Chao WS, Yeh HZ, Jan YG, Yen Y, Cheng SB, Wu CC, Lin YL, P'eng FK. 2019. Liver resection for hepatocellular carcinoma in oldest old patients. World J Surg Oncol 17(1):1 doi 10.1186/s12957-018-1541-0.

Xie X, Gao Y, Zeng M, Wang Y, Wei TF, Lu YB, Zhang WP. 2019. Nicotinamide ribose ameliorates cognitive impairment of aged and Alzheimer's disease model mice. Metab Brain Dis 34(1):353-366 doi 10.1007/s11011-018-0346-8.

Yoshino J, Baur JA, Imai SI. 2018. NAD+ Intermediates: The Biology and Therapeutic Potential of NMN and NR. Cell Metab 27(3):513-528 doi 10.1016/j.cmet.2017.11.002.

Zhou CC, Yang X, Hua X, Liu J, Fan MB, Li GQ, Song J, Xu TY, Li ZY, Guan YF, Wang P, Miao CY. 2016. Hepatic $\mathrm{NAD}(+)$ deficiency as a therapeutic target for non-alcoholic fatty liver disease in ageing. Br J Pharmacol 173(15):2352-68. doi 10.1111/bph.13513.

Zhou X, Li Y, Zhang X, Guan YY, Puentes Y, Zhang F, Speliotes EK, Ji L. 2019. Independent markers of non-alcoholic fatty liver disease in a gentrifying population based Chinese cohort. Diabetes Metab Res Rev 20:e3156 doi 10.1002/dmrr.3156. 
518 Figure 1 Effects of NR repletion on the body weight, food intake, relative liver weight and fat 519 pad mass of aged mice. Compared with the young, aged C57BL/6 mice were treated with NR 520 (400 mg/kg/day) for 3 consecutive months. (A) Effect of NR repletion on the body weight of 521 aged mice. (B) Effect of NR supplementation on the food intake of aged mice. Changes of 522 relative liver weight (C) and fat pat mass (D) caused by NR treatment. Values are mean $\pm \mathrm{SEM}$, 523 (n=5-6 per group). ${ }^{*} P<0.05,{ }^{* *} P<0.01,{ }^{* * *} P<0.001$ vs Young mice, ${ }^{\#} P<0.05$ vs aged mice.

524

525 Figure 2 Effect of NR repletion on the development of the NAFLD in aged mice. The levels of 526 triglyceride (A), total cholesterol (B), ALT (C), AST (D) and NAD (E) in serum of aged mice. F. 527 hepatic steatosis was reduced by NR administration in aged mice. The percentage of classified 528 livers in each of the four steatosis categories in different groups was as follows: 0, no 529 vacuolation; 1, 2 or 3 vacuoles; 2 , less than $50 \%$ of fatty vacuolation; 3 , more than $50 \%$ of fatty 530 vacuolation. (G-L) Representative images stained with H\&E of liver tissues of aged mice (400×; 531 scale bar, $50 \mu \mathrm{m}$ ); box regions are shown at higher magnification under the original pictures. 532 Values are mean \pm SEM, ( $\mathrm{n}=5-6$ per group). ${ }^{*} P<0.05 v s$ Young mice, ${ }^{\sharp} P<0.05 v s$ aged mice.

533

534 Figure 3 Effect of NR repletion on liver fibrosis in aging-relative NAFLD model. (A-C) 535 Masson's staining in livers from aged mice and (D) quantitative analysis. Red arrows show 536 positive blue staining for Masson. (E-G) Protein level of TGF- $\beta$ was detected by 537 immunohistochemistry and $(\mathrm{H})$ quantittive analysis. Red arrows show positive brown staining 538 for TGF- $\beta$. 200 $\times$; Scale bars, $100 \mu$ m. Values are mean \pm SEM, ( $\mathrm{n}=5-6$ per group). ${ }^{* *} P<0.01$, 539 *** $P<0.001$ vs Young mice, ${ }^{\#} P<0.05,{ }^{\# \#} P<0.001$ vs aged mice.

540

541 Figure 4 Effect of NR repletion on inflammatory infiltration of aging-relative NAFLD model.

542 (A-D) Immunohistochemistry staining in liver of aged mice showed the effect of NR 543 supplementation on Kupffer cell accumulation and (I) quantittive analysis. Red arrows show 544 positive brown staining for F4/80 or CD68. (E-H) Protein levels of IL-1 $\beta$ and TNF- $\alpha$ were 545 detected by immunohistochemistry and (J) quantittive analysis. Red arrows show positive brown 546 staining for IL-1 $\beta$ or TNF- $\alpha$. 200×; Scale bars, $100 \mu \mathrm{m}$. Values are mean \pm SEM, $(n=5-6$ per 547 group). $* P<0.05$. 


\section{Figure 1}

Effects of NR repletion on the body weight, food intake, relative liver weight and fat pad mass of aged mice

Compared with the young, aged C57BL/6 mice were treated with NR (400 mg/kg/day) for 3 consecutive months. (A) Effect of NR repletion on the body weight of aged mice. (B) Effect of NR supplementation on the food intake of aged mice. Changes of relative liver weight (C) and fat pat mass (D) caused by NR treatment. Values are mean \pm SEM, ( $n=5-6$ per group). ${ }^{*} P<0.05,{ }^{*} P<0.01,{ }^{* * *} P<0.001$ vs Young mice, ${ }^{*} P<0.05$ vs aged mice. 
A B
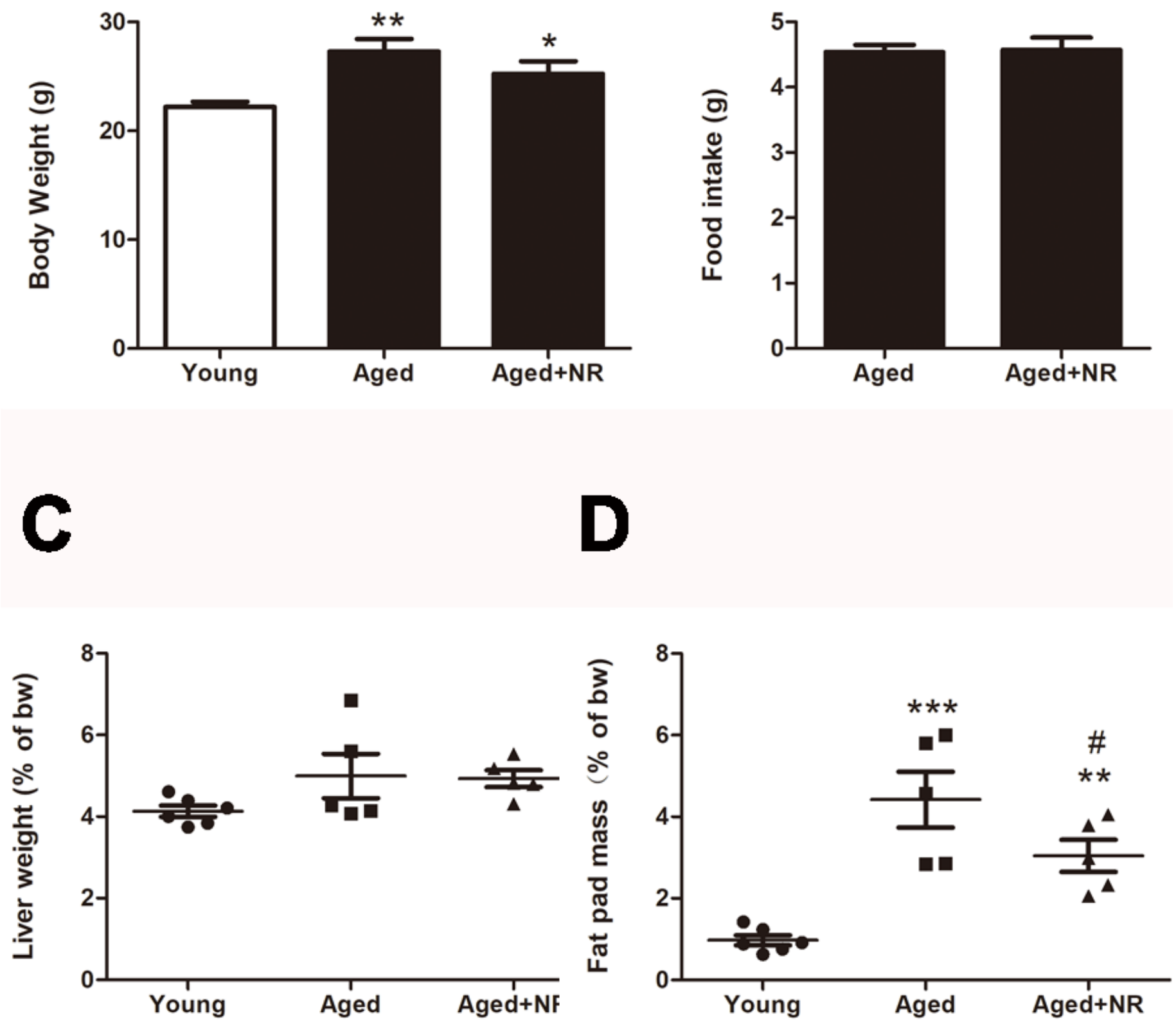


\section{Figure 2}

Effect of NR repletion on the development of the NAFLD in aged mice

The levels of triglyceride (A), total cholesterol (B), ALT (C), AST (D) and NAD (E) in serum of aged mice. F. hepatic steatosis was reduced by NR administration in aged mice. The percentage of classified livers in each of the four steatosis categories in different groups was as follows: 0 , no vacuolation; 1,2 or 3 vacuoles; 2 , less than $50 \%$ of fatty vacuolation; 3 , more than $50 \%$ of fatty vacuolation. (G-L) Representative images stained with H\&E of liver tissues of aged mice (400x; scale bar, $50 \mu \mathrm{m})$; box regions are shown at higher magnification under the original pictures. Values are mean \pm SEM, ( $n=5-6$ per group). $* P<0.05$ vs Young mice, ${ }^{\#} P<0.05$ vs aged mice. 
A

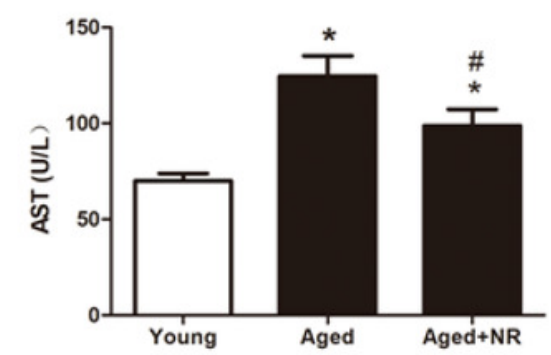

D

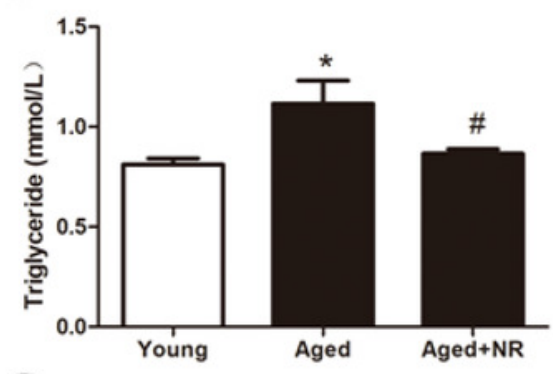

G

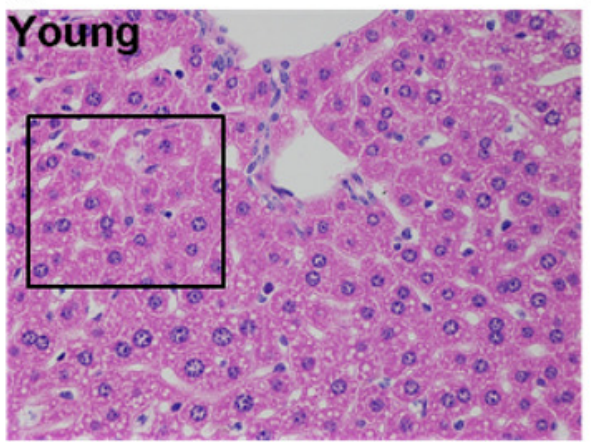

H

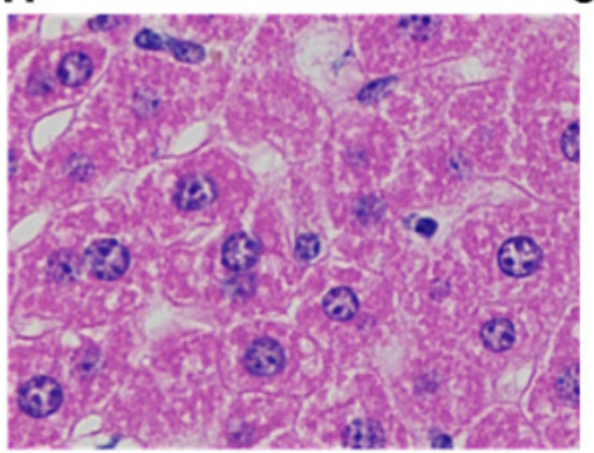

B

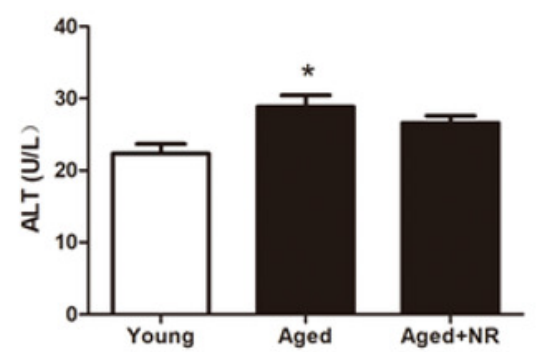

E

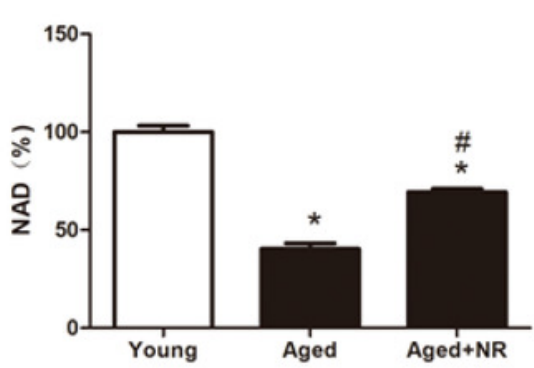

I

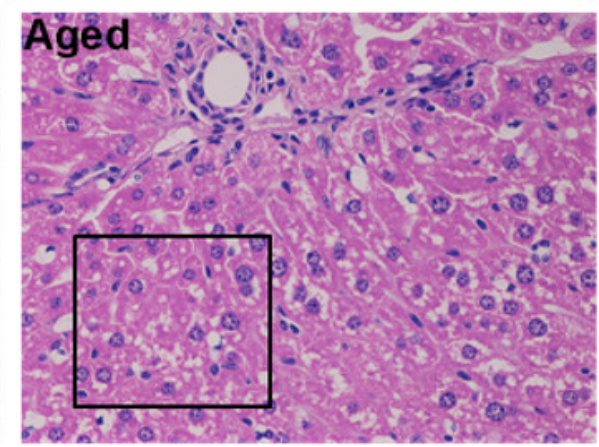
J

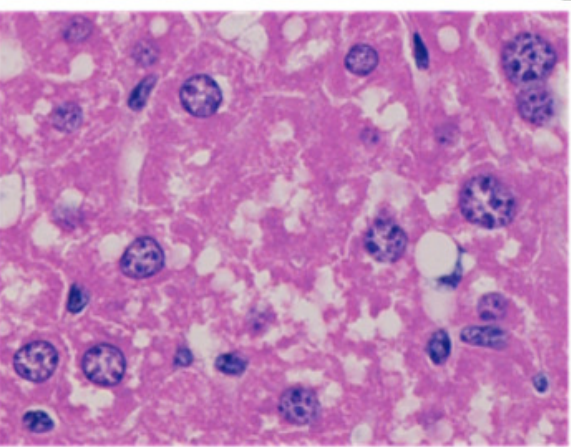

C

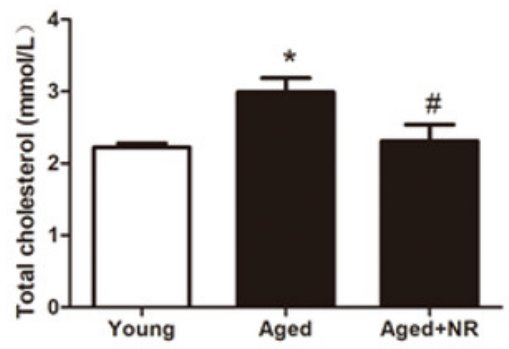

F

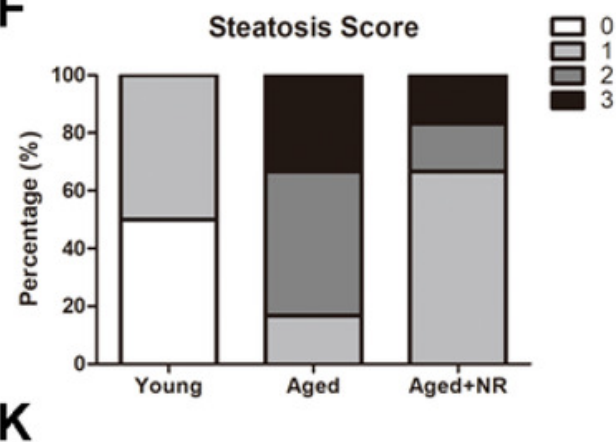

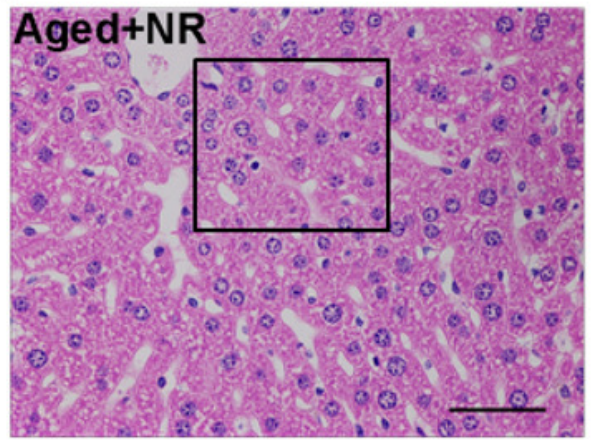

L

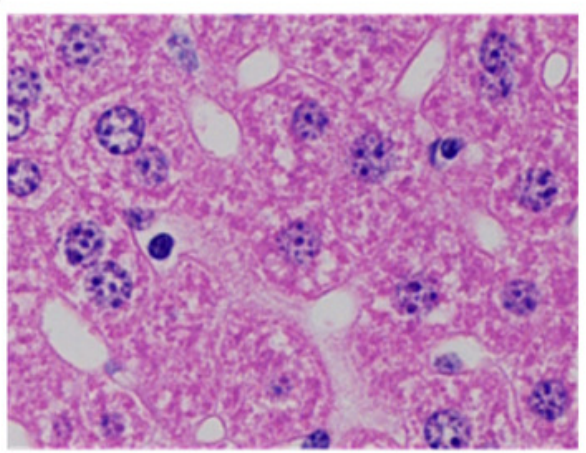




\section{Figure 3}

Effect of NR repletion on liver fibrosis in aging-relative NAFLD model

(A-C) Masson's staining in livers from aged mice and (D) quantitative analysis. Red arrows show positive blue staining for Masson. (E-G) Protein level of TGF- $\beta$ was detected by immunohistochemistry and $(\mathrm{H})$ quantittive analysis. Red arrows show positive brown staining for TGF- $\beta .200 x$; Scale bars, $100 \mu$ m. Values are mean \pm SEM, $\left(n=5-6\right.$ per group). ${ }^{* *} P<0.01$, ${ }^{* * *} P<0.001$ vs Young mice, ${ }^{\#} P<0.05,{ }^{\# \# \#} P<0.001$ vs aged mice.

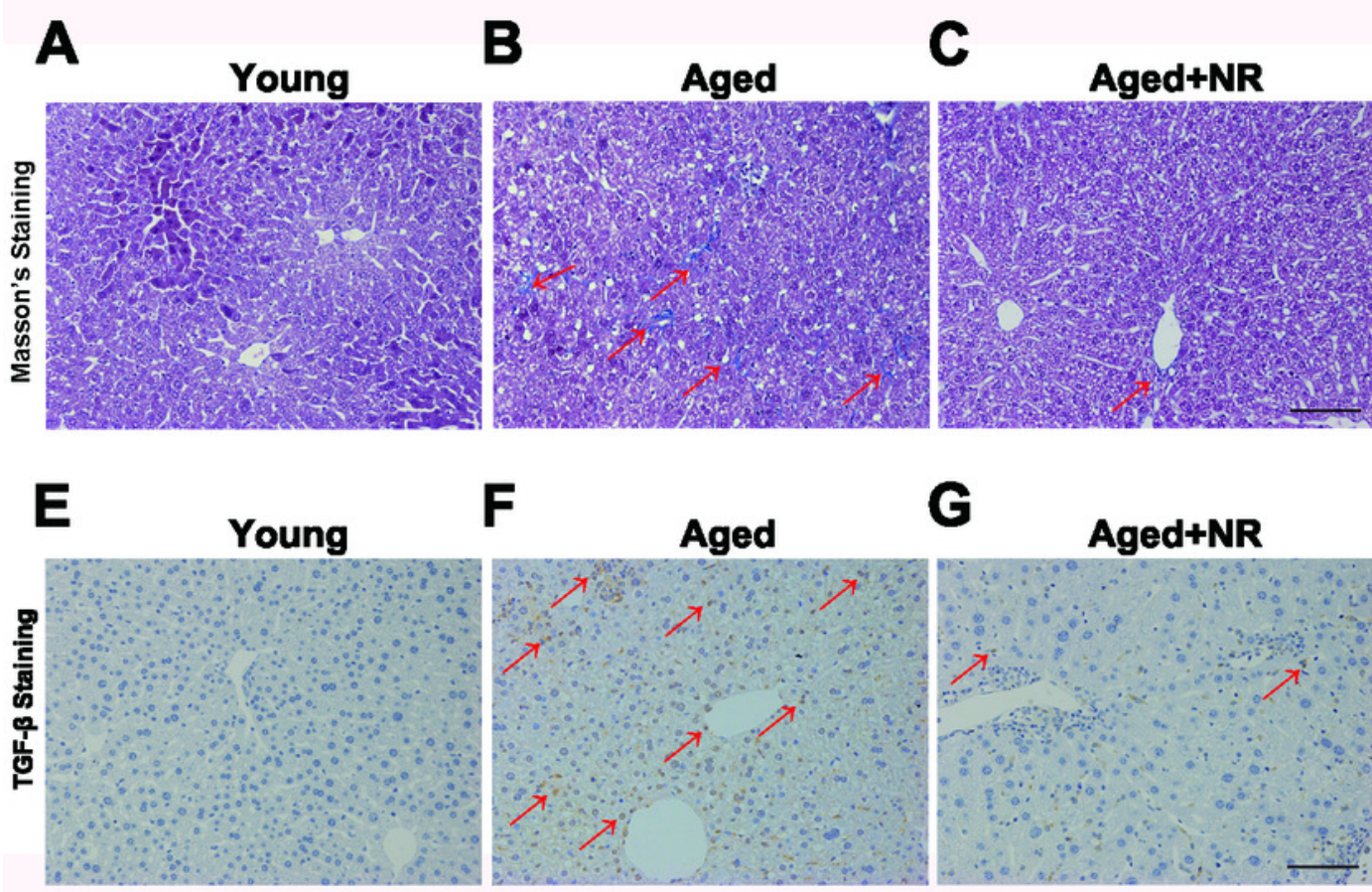

D

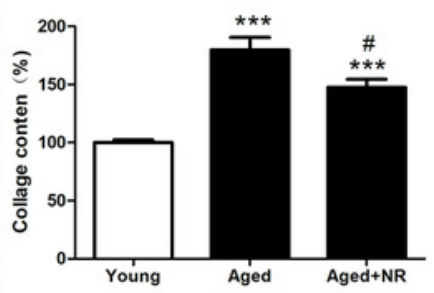

H

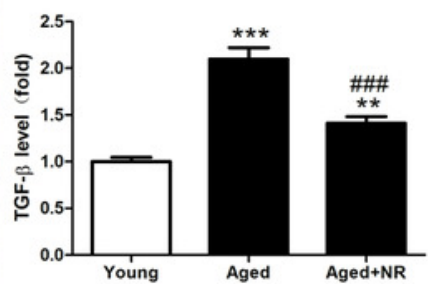




\section{Figure 4}

Effect of NR repletion on inflammatory infiltration of aging-relative NAFLD model

(A-D) Immunohistochemistry staining in liver of aged mice showed the effect of NR

supplementation on Kupffer cell accumulation and (I) quantittive analysis. Red arrows show

positive brown staining for F4/80 or CD68. (E-H) Protein levels of IL-1 $\beta$ and TNF- $\alpha$ were

detected by immunohistochemistry and (J) quantittive analysis. Red arrows show positive

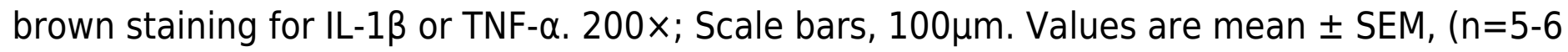
per group). $* P<0.05$. 

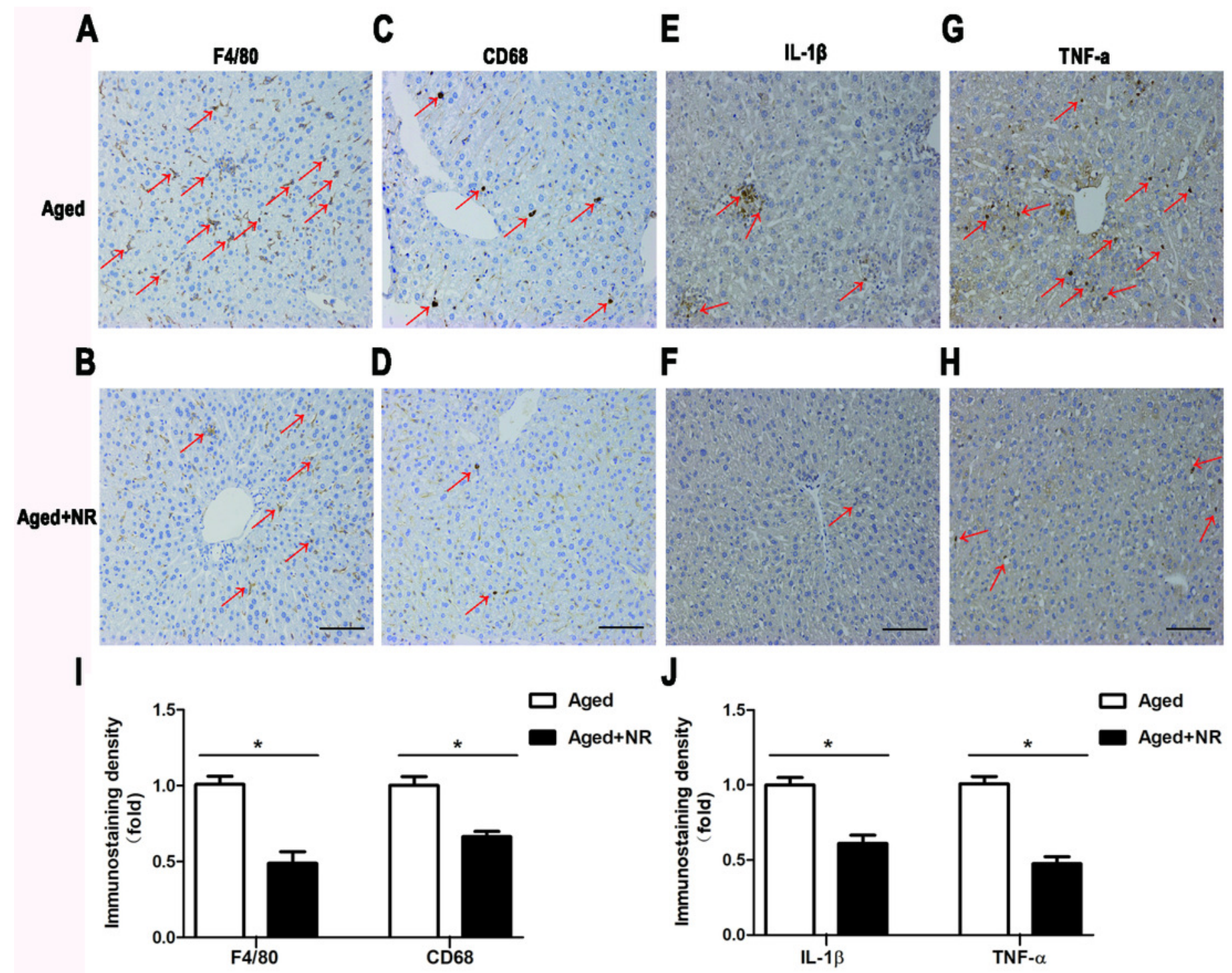

J

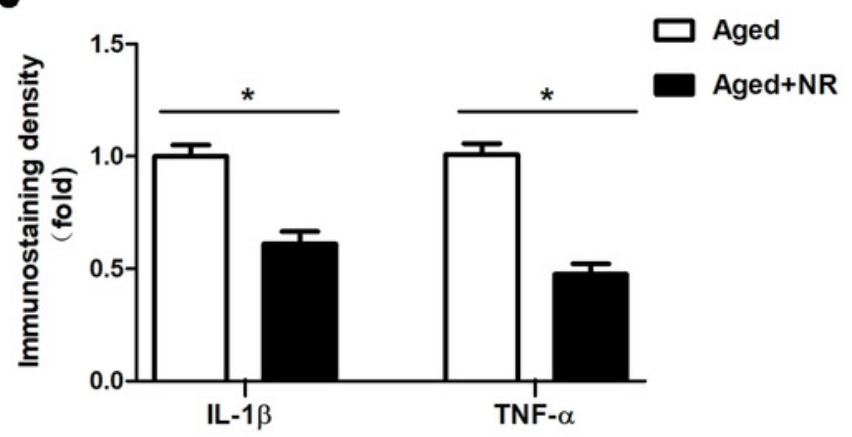

